\title{
Die schöne Transparenz-Norm und das Biest des Politischen: Paradoxe Folgen einer neuen Ideologie der Öffentlichkeit
}

Als Barack Obama seine erste Präsidentschaft antrat, versprach er » ein bisher nicht gekanntes Niveau an Öffentlichkeit der Regierung " ${ }^{1}$ und bewies damit treffsicheres Gespür für den Geist der Zeit. Als gelte es, eine bis in die Antike zurückreichende Vision besserer Politik nun mit den Mitteln des Internets endlich praktisch werden zu lassen, beobachten wir innerhalb der letzten Dekade eine regelrechte Welle von zivilgesellschaftlichem Transparenz-Aktionismus. Dicht auf den Fersen des sogenannten Web 2.0 $0^{2}$ entstand eine Reihe neuer Organisationen und Online-Plattformen, die sich mit Vehemenz für mehr Transparenz in Politik und Verwaltung einsetzen. Prominente Beispiele sind die »Sunlight Foundation « (gegründet 2006), die »Open Government Partnership « (2011), »Transparency and Accountability Initiative " (2012) oder in Deutschland " Mehr Demokratie e.V." (2003) und »Frag den Staat " (2011). Einige arbeiten wie "WikiLeaks « (2006) konfrontativ, andere bereiten öffentlich zugängliche Informationen auf wie etwa »Abgeordnetenwatch.de « (2004), wieder andere drängen eher auf institutionelle Reformen wie das "Government 2.0 Netzwerk Deutschland « (2009), konkret in Gestalt von Informationsfreiheits- und Transparenz-Gesetzen. Die Politik hat sich diese Forderungen teils schon zu eigen gemacht, was sich neben dem Beispiel aus den USA unter anderem daran zeigt, dass der deutsche Bundesbeauftragte für Datenschutz seit der Herausgabe seines 21. Tätigkeitsberichts (2007) als Bundesbeauftragter für Datenschutz und Informationsfreiheit auftritt.

Die Philosophie der Transparenz-Akteure kreist stets um dieselben Schlagworte, wie ein Blick auf ihre Internetauftritte zeigt: Es werden Offenheit und Transparenz von Regierung und Verwaltung durch möglichst freien Informationsfluss gefordert. Dies führe, so wird unterstellt, zu einer Stärkung der Rechenschaftspflicht der Regierung, mehr demokratischer Partizipation, einer Ermächtigung der Zivilgesellschaft und mehr Vertrauen. "Information bedeutet Macht «, ${ }^{3}$ heißt es in vielen einschlägigen Selbstdarstellungen. Je mehr Macht beim Volk liegt, so die Prämisse, desto näher komme die Demokratie ihrem Ideal.

1 Obama White House Memorandum: Transparency and Open Government. www.whiteh ouse.gov/the_press_office/TransparencyandOpenGovernment

(Zugriff vom 13.05.2014).

2 Auch »social media « genannt, vgl. Egger de Campo 2014.

3 Sunlight Foundation: Our Mission. https://sunlightfoundation.com/about/ (Zugriff vom 25.04.2014). 
Die eigentliche Triebfeder für mehr Transparenz in der Politik (und vielleicht auch den Transparenz-Aktivismus selbst) ist jedoch das Internet. Das Internet gilt als »Transparenzmedium schlechthin ${ }^{4}$. Informationsfreiheitsgesetze nehmen nur wenige Menschen in Anspruch, aber das Internet setzt, indem es die Grenzen von Raum und Zeit sprengt, enorme Informationsmengen frei. Jede registrierbare Bewegung der Politik wird im Netz aufgezeichnet, und jede(r) Bürger(in) kann, vermittelt durch die Medien oder unter bewusster Umgehung etablierter Kanäle, über online verfügbare Quellen tiefe oder zumindest direkte Einblicke in das politische Geschehen nehmen. Wir leben heute, so David Runciman, in »einem Zeitalter fast unbegrenzter Möglichkeiten der Beobachtung der politischen Klasse, jenseits all dessen, was Jeremy Bentham sich erträumt oder auch erwünscht haben mochte «. ${ }^{5}$

Es gibt viele Gründe, sich mit der neuen Transparenz-Bewegung zu befassen. Hier sollen zwei in den Vordergrund gerückt werden. Zum einen schwingen im Begriff der Transparenz respektable liberale Prinzipien mit: dass nämlich eine Regierung demokratischer Kontrolle zu unterwerfen ist und ihr nur genau so viel Macht zusteht, wie es der kollektiven Selbstbestimmung mündiger Bürger entspricht. Mehr nicht. Diese demokratische Selbstbestimmung vollzieht sich im Modus der räsonierenden, zur Vernunft begabten liberalen Öffentlichkeit. Zum anderen scheinen die praktischen Entwicklungen uns diesem Ideal - ganz entgegen den Vorstellungen des Transparenz-Aktivismus - nicht näherzubringen: Bei steigender Transparenz hat das Vertrauen in die Politik in den letzten Dekaden eher abgenommen. In den USA, die faktisch wohl eines der transparentesten Regierungssysteme haben (erstaunlicherweise gibt es noch keine international-vergleichende Transparenz-Rangliste ${ }^{6}$ ), hat sich seit dem Amtsantritt von Obama infolge heftigen parteipolitischen Streits eine regelrechte Politikverachtung breitgemacht. Transparenz ist zu einer globalen Demokratienorm geworden, hat aber bislang keinen weiteren Demokratisierungstrend angeschoben. Skeptisch stimmt auch, dass gerade die politische Elite selbst, die durch Transparenz demokratischer Kontrolle unterworfen werden soll, sich vielfach den Enthusiasmus der Aktivisten zu eigen macht.

Ist die Transparenz-Bewegung also eine Ideologie, die vielleicht mehr von den Möglichkeiten des Internets inspiriert ist als von tatsächlichen Problemen der modernen Demokratie? Die Politikwissenschaft bezweifelt mittlerweile, besonders im englischsprachigen Raum, ob Transparenz tatsächlich »der Schlüssel zu einer besseren Demokratie « 7 ist. Um nur die wichtigsten Einwände anzureißen: Politik und Verwaltung fänden stets Mittel und Wege, um sich Transparenz-Anforderun-

\section{Zehnpfennig 2013, S. 46.}

5 Runciman 2008, S. 199.

6 Allerdings gibt es ein Länderranking vom Centre for Law and Democracy und von Access Info Europe in Bezug auf Informationsfreiheitsgesetze (www.rti-rating.org/country_rating .php; Zugriff vom 08.07.2014). Das Ranking berücksichtigt nur den de jure-Zustand und führt daher zu kuriosen Ergebnissen: Einige nichtdemokratische Staaten wie Aserbaidschan, Jemen und Äthiopien liegen ganz oben, mehrere westliche Demokratien ganz unten; von den 89 untersuchten Staaten ist Österreich (sic!) das Schlusslicht.

7 Hood, Heald 2006; vgl. Shane 2004; O’Neill 2002.

Leviathan, 42. Jg., 3/2014 
gen zu entziehen; ${ }^{8}$ dies zeige sich besonders an der Sicherheitsgesetzgebung infolge der Terrorismusbekämpfung; ${ }^{9}$ Transparenz könne auch autokratische Regime stützen, wenn diese kompetent seien; ${ }^{10}$ sie konkurriere mit anderen Werten wie Effektivität, Effizienz, Legitimität etc. und könne daher kein absolutes Ziel sein; ${ }^{11}$ Bürokratien, nach Max Weber die Leistungsträger der modernen Politik, würden unter vollständiger Transparenz nicht funktionieren; ${ }^{12}$ und Transparenz verschärfe das klassische demokratietheoretische Problem noch, dass mehr Information eben kein besseres politisches Urteil bedeute. ${ }^{13}$

An diesen Diskurs schließe ich hier mit einer grundsätzlicheren Überlegung zu den illusorischen Versprechen von Transparenz an. Ich teile Göttrik Wewers Auffassung, dass die Forderung nach Transparenz mehr »aus dem Bauch [kommt], nicht sonderlich reflektiert und schwerlich geeignet [ist], die Demokratie zu stärken « ${ }^{14}$ Eine Norm, welche in das komplizierte Gefüge des demokratischen Prozesses eingreift, sollte einer sorgfältigen Wirkungsanalyse unterzogen werden. Die Hypothese lautet: Ein Übermaß an Transparenz transformiert nicht die Politik zum Besseren, sondern akzentuiert im Gegenteil noch jene Mechanismen, die gemeinhin als undemokratisch (miss)verstanden werden. Machtgefälle und Interessengegensätze werden nicht zu Gunsten einer partizipativen und deliberativen Politik überwunden, sondern unter dem Anschein einer offenen Regierung eher noch verstärkt. Mit anderen Worten: Die Beharrungskraft des Politischen könnte sich als stärker erweisen als die Heilkraft der Transparenz.

\section{Liberale Wurzeln der Transparenz-Norm}

Die Transparenz-Kritik wandelt auf schmalem Grat, denn Transparenz ist nicht nur eine intuitiv angemessene, moralische Universalnorm. So schrieb etwa Rousseau: »Ein einziges Gebot der Sittenlehre kann aller andern Stelle vertreten, dieses nämlich: Tue und sage niemals etwas, was nicht die ganze Welt sehen und hören könnte. Ich meinerseits habe stets jenen Römer als den hochachtungswürdigsten Mann betrachtet, der wünschte, sein Haus werde so gebaut, daß man alles, was darin vorginge, sehen könnte «. ${ }^{15}$ Transparenz ist, angewandt auf die Regierung, auch eine Gründungsnorm der Demokratie. »Die Gesellschaft hat das Recht, von jedem Staatsbeamten Rechenschaft über seine Amtsführung zu verlangen ", so bereits die Erklärung der Menschen- und Bürgerrechte von 1789 (Artikel 15), und praktisch bedeutet dies, die entsprechenden Informationen erlangen zu können. Der Staat soll

8 Roberts 2006 b.

9 Roberts 2006 a.

10 Lord 2006, S. 96.

11 Heald 2006.

12 Wewer 2014.

13 Zehnpfennig 2013, S. 46-48.

14 Wewer 2014, S. 6.

15 Zitiert nach Han 2012, S. 72. 
keine höfische Arkanpolitik mehr treiben dürfen, sondern steht in Diensten seiner Bürger, des Souveräns. Ideengeschichtlich lassen sich nun zwei, bei genauer Betrachtung nicht ganz spannungsfreie Funktionen von Transparenz in der Beziehung von Staat und Bürger ausmachen, nämlich (a) Kontrolle durch Sichtbarkeit und (b) Mitbestimmung durch die liberale Öffentlichkeit.

(a) Transparenz konstituiert Öffentlichkeit und unterwirft damit das Verhalten den Normen des Gemeinwesens. »Das Licht der Sonne ist das beste Desinfektionsmittel, elektrisches Licht der beste Polizist. «16 Die Rede vom Polizisten lässt erkennen, dass diese Art von Kontrolle sich in den Anfängen der Demokratie zunächst auf das Volk richtete. Jeremy Benthams häufig zitierte Idee des Panoptikums, heute mit Totalitarismus assoziiert, wurde im Zusammenhang seiner Lehre des Utilitarismus entworfen, die ein Versuch war - eigentlich eine Form des »social engineering " -, eine für die moderne Industriegesellschaft angemessene Moral zu finden. In seiner architektonischen Manifestation war das Panoptikum ein Instrument, um die Menschen im Gefängnis zu disziplinieren, in der Anwendung auf andere gesellschaftliche Einrichtungen aber ein Prinzip, um die Menschen zu vernünftigem Verhalten im Sinne utilitaristischer Prinzipien zu disponieren. Die ungeheure Effizienz des Panoptikums beruht auf einer Asymmetrie: Dem Beobachter ist alles transparent, er selbst ist jedoch für den Beobachteten unsichtbar. Die Möglichkeit permanenter Beobachtung bewirkt, dass äußere Kontrolle in eine innere Selbstkontrolle transformiert wird. Entscheidend ist nun, dass Bentham in seiner späteren Schaffensphase, enttäuscht von der Politik, die Beobachtungsrichtung auf die Regierung umlegte $^{17}$ und damit ein früher Vorläufer der Idee vom »gläsernen Staat « wurde. Wenn jeder Verwaltungsakt und jede politische Tat potenziell öffentlich und damit durch Wahlen, gerichtliche Anfechtung oder die öffentliche Meinung etc. sanktionierbar sind, müssen Politik und Verwaltung ein äußerstes Maß an vorauseilender Gemeinwohlorientierung üben.

Das Internet selbst gemahnt von seiner Struktur her an ein Panoptikum: Es ermöglicht über Raum und Zeit hinweg und womöglich noch mit Hilfe der verschiedenen Transparenz-Plattformen umfassende Einblicke in Politik und Verwaltung. Jede Normabweichung kann, irgendwo registriert und für alle online dokumentiert, in zukünftigen und völlig unabsehbaren Kontexten zum Skandal explodieren. Undemokratisches Verhalten wird davon - jedenfalls in der Theorie - schon im Keim erstickt, selbst in jenen Bereichen, etwa der Geheimdienstarbeit, die vor der Gründung von WikiLeaks recht gut abgeschirmt waren gegenüber öffentlicher Kontrolle.

(b) In der zweiten Funktion wird Kontrolle weniger konfrontativ, sondern konstruktiv ausgeübt. Immanuel Kant und John Stuart Mill sind die klassischen Philosophen der »liberalen Öffentlichkeit «18, aber am weitesten entfaltet - und im heutigen Internetdiskurs am häufigsten aufgegriffen - finden sich diese Überlegungen bei Jürgen Habermas. Auch er teilt die Vorstellung, dass Macht und Herrschaft auf

16 Siehe www.law.louisville.edu/library/collections/brandeis/node/196 (Zugriff vom 08.07.2014).

17 Rzepka 2013, S. 63-124.

18 Vgl. Zehnpfennig 2013.

Leviathan, 42. Jg., 3/2014 
eine neue Grundlage gestellt werden können. Eine Politik im Modus von Öffentlichkeit, Vernunft und Wahrheit wird für prinzipiell möglich gehalten. Das historische Ideal für die liberale Öffentlichkeit erblickt Habermas in der bürgerlichen Öffentlichkeit der Kaffeehaus-Diskussionen des 18. Jahrhunderts, in denen mündige Privatbürger über Politik debattieren und damit zu Subjekten des Staats werden. Seine später in Faktizität und Geltung ${ }^{19}$ entworfene Theorie der »deliberativen Politik « formuliert das historische Ideal zu einem nun überwiegend kontrafaktischen, normativen Entwurf um. Öffentlichkeit wird politisch wirksam, indem ein räsonierendes zivilgesellschaftliches Publikum Themen in der Öffentlichkeit verankert und debattiert, welche dann in den Bereich der institutionalisierten Politik hineinwirken. Die klassische Macht- und Interessenpolitik im Zentrum wird von der Entfaltung kommunikativer Macht in der diskursiv erstarkten, zivilgesellschaftlichen Peripherie eingekreist. Diese Einkreisung beziehungsweise das rationale Potenzial einer deliberativen Politik ist umso stärker, je mehr der Diskurs den Kriterien einer »idealen Sprechsituation « entspricht: Machtfrei soll er sein, möglichst inklusiv, und die Sprecher sollen bereit sein, ihr partikulares Interesse vom Allgemeinwohl her zu rechtfertigen.

Der Begriff »Transparenz « findet sich bei Habermas nicht, kann aber als Prämisse eines gelingenden, an den drei »Geltungsansprüchen « Wahrheit, Angemessenheit und Aufrichtigkeit orientierten Diskurses ergänzt werden. Die Demokratieforschung hat Habermas und das Internet denn auch schnell zusammengeführt. ${ }^{20}$ Die Formulierung von Witschge beschreibt dabei den Ausgangspunkt dieses Forschungsparadigmas sehr gut: Das Internet »bringt uns der Lösung von vier Problemen näher, die volle Partizipation in modernen Demokratien schwierig, wenn nicht unlösbar machten: Zeit, Größe, Wissen und Zugang [zum politischen Diskurs] «. ${ }^{21}$ Bislang steht die Erwartung einer mehr direkten und deliberativen Politik, empirisch betrachtet, aber noch auf schwachen Beinen.

\section{Problematische Prämissen von Transparenz}

Dem deliberativ-partizipativen Politikmodell liegen problematische Prämissen zugrunde, etwa was die komplementäre Rolle der Bürger und die Existenz strategischer Interessen betrifft. Bevor jedoch diese Spur aufgenommen wird, möchte ich kurz bei einer anderen Prämisse verweilen, die für die Transparenz-Aktivisten einen besonderen Reiz zu haben scheint. Gemeint ist eine Art doppelte Einheitsprätention, nämlich in rational-intelligibler und sozialer Hinsicht.

Vernunft als Modus der Politik impliziert, dass es das "Vernünftige " gibt, worauf sich vernunftfähige Individuen einigen können. So wurde in den frühen Konzeptionen der liberalen Öffentlichkeit und noch bei Kant angenommen, dass es eine naturrechtlich vorbestimmte oder anderweitig ideale gesellschaftliche Ordnung gäbe.

19 Habermas 1992.

20 Vgl. zum Beispiel Leggewie, Maar 1998; Plake et al. 2001; Shane 2004.

21 Witschge 2004, S. 110. 
Politik bedeutet in diesem Konzept "Wahrheitsfindung im Prinzipiellen ", nicht »Willensbildung « ${ }^{22}$ Habermas selbst rechnet sein Modell der deliberativen Politik zwar einer Theorie der Willensbildung zu, jedoch bleibt bei ihm die Vorstellung einer konsensualen, das heißt an soziale Bedingungen geknüpften Wahrheit leitend. Interessengegensätze werden durch den Konsens nicht eliminiert, aber die Anhänger der deliberativen Demokratie unterstellen, dass sie sich durch ein rational motiviertes Einverständnis vom Gemeinwohl her entschärfen lassen. Rationalität, begünstigt durch Informationen, hätte demnach eine Tendenz zum demokratisch »richtigen « Resultat.

Dem entspricht, zweitens, die Prätention einer Einheit von Gesellschaft und Politik, von Bürgern und ihren Repräsentanten. Das deliberative Politikmodell konzipiert die Nation nach dem Bild der griechischen Agora als inklusiv und egalitär. ${ }^{23}$ Die Agora gelte, so Kamps, als »Inbegriff und Sinnbild einer effizienteren und wohl auch emotional befriedigenderen Bindung der Bevölkerung an die Politik - und umgekehrt «. ${ }^{24}$ Im Politikmodell der Piraten, der »liquid democracy «, wurde der Versuch unternommen, jede Art von Repräsentation, die wohl als Quelle für demokratische Störungen gilt, zu beseitigen. »Die >Piratenpartei< [...] ist ihre Wählerschaft. « ${ }^{25}$ Begriffe wie "gläserner Staat " und "gläserner Abgeordnete " eliminieren semantisch die Differenz von Staat und Volk. Das Prinzip der Repräsentation, institutionalisiert durch Wahlen, geht über in das Prinzip Identität. In der Folge bezieht sich die Kontrolle nicht mehr auf das politische Programm, sondern setzt bei der Person und ihren Motiven an. Dies äußert sich zum Beispiel in der Diagnose, dass mangelndes Vertrauen ein Problem der heutigen Demokratie sei. Vertrauen avanciert zum neuen Code der demokratischen Öffentlichkeit, entsprechend der Rolle von Geld in der Wirtschaft. Es wird im Einzelfall bereitwilliger gegeben, aber deswegen auch schmerzlicher enttäuscht - mit Folgen für das Vertrauen in die Demokratie insgesamt.

Das Gegenteil dieser antike Ideale reflektierenden Konzeption der Demokratie wäre ein Begriff des Politischen, dessen Grundkategorien Macht, Interesse, Wille und Pluralismus sind. In der Demokratietheorie selbst finden sich dazu keine Anknüpfungspunkte, deuten diese Begriffe doch in eher nichtdemokratische Richtungen. Was gemeint ist, ließe sich jedoch zum Teil aus der Kritik an Habermas rekonstruieren, ${ }^{26}$ zum Teil aus älteren und kritischen Beiträgen der politischen Theorie: John Stuart Mill fürchtete wie schon Alexis de Tocqueville die »Tyrannei der Mehrheit «, ${ }^{27}$ weshalb die rohe Kraft der Öffentlichkeit durch Repräsentation, Rechtsstaat und Gewaltenteilung beschränkt werden müsse; Max Webers Theorie des Dezisionismus unterstellt, dass konsensuale Entscheidungen in einer fragmen-

22 Vgl. Habermas 1962, S. 145.

23 Young 1996, S. 125-126; König 2012, S. 23.

24 Kamps 2000, S. 228.

25 Zehnpfennig 2013, S. 52.

26 Vgl. Benhabib 1996.

27 Mill 2010 [1859], S. 11. 
tierten Gesellschaft nicht mehr möglich sind, was bei Carl Schmitt wiederum zur Freund-Feind-Unterscheidung als Natur des Politischen gesteigert wird; die kritische Politiktheorie von Chantal Mouffe kreist um die Grundbegriffe Macht und Konflikt. ${ }^{28}$ Zwar übertreiben diese Ansätze in die andere Richtung eines kampfbereiten Heroismus, ${ }^{29}$ aber sie warnen uns jedenfalls davor, Politik in allzu harmonischen Farben zu sehen. Die Väter der modernen westlichen Verfassungen haben diese Warnung beherzigt: Der repräsentative Rechtsstaat ist durch zahlreiche Prinzipien des institutionalisierten Misstrauens ausgezeichnet - Gewaltenteilung, Opposition, Misstrauensvotum etc. ${ }^{30}$ Das Grundgesetz beruht eher auf der Annahme, dass Misstrauen und kritische Distanz, nicht aber Vertrauen die angemessene Haltung gegenüber dem Staat sind. ${ }^{31}$

\section{Transparenz und die anspruchsvolle Komplementärrolle des Bürgers}

Transparenz fördere die Informationsgesellschaft, so Ingolf Pernice vom Institut für Internet und Gesellschaft, ${ }^{32}$ und damit werde $»$ Politik wirklich Sache der Bürger «. ${ }^{33}$ In diesem Sinne hatte sich auch die Enquete-Kommission »Internet und Gesellschaft « zum Ziel gesetzt, » die Öffentlichkeit in den Arbeitsprozess so intensiv wie möglich einzubeziehen ", unter anderem durch das Live-Streaming der Sitzungen und durch eine Online-Bürgerbeteiligung über die Software »Adhocracy «. ${ }^{34}$ Hieran wird eine Implikation der Transparenz-Programmatik deutlich, die von den Aktivisten kaum hinreichend reflektiert wird: Offensichtlich richtet sich die Forderung nach Transparenz nicht allein an den Staat, sondern sie enthält stets auch eine gegenläufige Erwartung an die Bürger. Denn sie erfüllt ihre Wirkung erst dann, wenn die Bürger ihre komplementäre Rolle von kritischer Beobachtung und aktiver Mitwirkung zu spielen wissen. Dem Staat durch Transparenz Macht und Verantwortung abzuringen ergibt daher nur Sinn, wenn die Bürger zur Aufnahme derselben bereit sind.

Die Anforderungen an die Bürger sind hoch, denn moderne Gesellschaften und ihre Politik sind komplex. Zu Lebzeiten von Jeremy Bentham (1745-1832), der

28 Mouffe 2007.

29 Diese Konzepte neigen dazu, den »Moment « und die "politische Haltung « beziehungsweise die Aktion zu betonen statt jene Strukturen - Rechtsstaat, Gewaltenteilung, Repräsentation -, welche die Freiheit der Bürger und den gesellschaftlichen Interessenausgleich sichern (Volk 2013, S. 93, 97).

30 Sztompka 2010, S. 285-287.

31 Manent 2014, S. 132.

32 Das Institut wurde 2012 von der Humboldt-Universität zu Berlin, der Universität der Künste Berlin und dem Wissenschaftszentrum Berlin für Sozialforschung gegründet, außerdem ist das Hans-Bredow-Institut für Medienforschung Kooperationspartner. Finanziert wird es von Google.

33 Pernice 2013, S. 14.

34 Enquete-Kommission 2013, S. 5. 
Transparenz als Mittel »einsehbarer Eindeutigkeit « 35 in der Politik für möglich und sinnvoll hielt, betrug die Staatsquote in Großbritannien etwa 10 Prozent, ${ }^{36}$ heute liegt sie in den modernen Industriestaaten bei rund 50 Prozent. Dementsprechend kann eine Potenzierung der politischen Komplexität angenommen werden. Die Steuerung moderner Gesellschaften wäre ohne eine leistungsfähige, hoch spezialisierte Bürokratie und Ministerialverwaltung, die bei vielen Themen nahe dem Stand des wissenschaftlichen Fortschritts operiert, gar nicht möglich. Dabei enthält jede Regulierung, auch die scheinbar trivialste Form der Kommunalpolitik, eine "autoritativ verfügte Verteilung von Werten" (so die bekannte Politikdefinition von David Easton), die Gewinner und Verlierer sowie Auswirkungen auf das Gemeinwohl hat. Umgekehrt müssen auch größere, parteipolitische Streitfragen von regulatorischem Sachverstand begleitet werden. An der Komplexität der Politik führt also kein Weg vorbei.

Kann Transparenz das daraus resultierende »Unbehagen an der Politik « ${ }^{37} \mathrm{~min}$ dern, und was wäre dabei die Rolle des Bürgers? Transparenz ist zunächst einmal eine merkwürdige Metapher: Der transparente Körper wird, insofern er durchsichtig ist, selbst unsichtbar. Er verschwindet im Blick des Betrachters. Genau dies könnte die praktische Wirkung von Transparenz in der Politik sein. Schuld ist ein epistemologisches Paradoxon: Je schärfer ein Detail betrachtet wird, desto mehr geraten die Strukturen aus dem Blick. Im Rasterelektronenmikroskop erkennt man Atome, aber nicht mehr, welches lebensweltliche Objekt sie bilden. So müssen die politischen »Rohdaten ", welche durch Transparenz ans Licht kommen, erst wieder zu politischem Sinn kombiniert werden - ein anspruchsvoller Prozess der Kontextualisierung, bei dem einiges schiefgehen kann.

Zum einen werden Zweifel angemeldet, ob der Bürger überhaupt die Zeit, das Interesse und die Kenntnisse hat, um Informationen, die ihm prinzipiell zugänglich sind, gezielt nachzufragen und bewerten zu können. ${ }^{38}$ Komplexe EU-Regulierung ist zum Beispiel in der Regel nicht Gegenstand privater Nachforschungen, verständlicherweise - wer kennt sich etwa mit dem Aktienmarkt so gut aus, dass er eine 500seitige Regulierung in Bezug auf den eigenen Pensionsfonds richtig einordnen könnte? Unter dem Gesichtspunkt des linearen Demokratiegewinns durch bessere Information der Bürger müssen Informationsfreiheitsgesetze als Fehlschlag gewertet werden. ${ }^{39}$ Damit versagt allerdings auch der von Bentham formulierte Kontrollmechanismus durch vorauseilende Selbstkontrolle: Wenn die Informationsflut derart anschwillt, dass eine Entdeckung politischer Normverstöße nicht mehr zu befürchten ist, greift das Prinzip Öffentlichkeit ins Leere. ${ }^{40}$ Bei voller Transparenz wird die Politik in gewisser Weise also eher opak.

35 Rzepka 2013, S. 105.

36 Schremmer 1994, S. 58.

37 Vgl. Oelze 2013.

38 Roberts 2006 a, S. 217.

39 Wewer 2014, S. 12.

40 Vgl. Rzepka 2013, S. 117-119. 
Es ist vor diesem Hintergrund bemerkenswert, wie Transparenz-Aktivisten dennoch auf Information als demokratisches Heilmittel setzen und wie wenig dabei ältere Einsichten der Demokratie- und Bildungsforschung berücksichtigt werden. Schon Giovanni Sartori weist in ausführlicher theoretischer Behandlung auf das eben beschriebene Problem hin, dass nämlich von Information alleine keine Steigerung bürgerlicher Rationalität zu erwarten sei: »Bei gleicher Informationsmenge ist jemand sachkundig oder nicht sachkundig, je nachdem, ob er richtig erkennt, welche Mittel welchen Zwecken dienlich sind, und somit, welche Folgen aus welcher Entscheidung oder Handlung erwachsen «. ${ }^{41}$ Information muss in Wissen und Wissen muss in politisches Urteil überführt werden, wofür wiederum Vorwissen erforderlich ist. Wir sprechen also von einem regelrechten Erkenntnisprozess.

Entsprechend mag auch die empirische Bildungsforschung, sofern sie sich des Themas überhaupt annimmt, keine Verbesserung infolge der Verbreitung des Internets und der Steigerung politischer Transparenz erkennen. Maier und Bathelt beklagen, dass zwar politische Einstellungen seit langem und recht intensiv erhoben würden, zum Beispiel in Umfragen wie dem Eurobarometer, aber nicht das politische Wissen der Bürger, in dem sich Bürgerkompetenz abbilden müsste. Die wenigen Studien, die es gibt, zeigten, dass »sich die Kenntnisse der Bürger über zentrale institutionelle Strukturen, politische Prozesse, Personen oder Themen in engen Grenzen halten « und über die Zeit hinweg auch »keine systematische Verbesserung des Wissensstandes auszumachen [ist] «. ${ }^{42}$ Zwar forscht die Kommunikationswissenschaft intensiv zu dem Thema und beschreibt eindrücklich, wie sich die Mediennutzung verändert, ins Internet verlagert und neue politische Kommunikationsformen entstehen. Auf dieser Grundlage wird dann enthusiastisch eine "neue Rolle der Bürger in der Politik « behauptet, ${ }^{43}$ was suggeriert, dass Transparenzgewinne in demokratische Qualitätssteigerungen umgewandelt werden könnten. Die aufwendige Studie von Maier und Bathelt zeigt jedoch entgegen der vorherrschenden Meinung, dass sich ausgerechnet »das Internet signifikant negativ [!] auf die politische Expertise ${ }^{44}$ auswirkt. Lediglich die Nutzung klassischer Printmedien ist, abgesehen vom Faktor Bildung, positiv mit politischem Wissen korreliert. Andere Studien zeigen, dass seit einer Dekade sich gerade die jüngere, internetaffine Generation der unter 30-Jährigen aus der öffentlichen Sphäre zurückzieht. ${ }^{45}$

Dieser Befund lässt zwar die Möglichkeit offen, dass Bürger das Internet für fallund themenspezifische Hintergrundrecherchen und die Artikulation begründeter Meinungen nutzen. Schließlich lässt die Netzgemeinde immer wieder einmal ihre Muskeln spielen und Proteststürme durch die Politik fegen, etwa in Form von Online-Petitionen. Allerdings ist bei insgesamt sinkendem Rationalitätsniveau fraglich, was die Quellen derartiger Eingaben sind. Sie dürften eher in den Empörungs-

41 Sartori 1992, S. 129.

42 Maier, Bathelt 2013, S. 415.

43 Vgl. Emmer 2013.

44 Maier, Bathelt 2013, S. 427.

45 Rentsch, Mothes 2013, S. 73-74. 
und Erregungswellen einer virulenten Netzöffentlichkeit als in einem kognitiv gefestigten politischen Engagement der Teilnehmer liegen.

Was ist der Grund? Es könnte mit einer Informationsüberflutung (information overload) und -inflation zu tun haben. Jede Information wirft potenziell neue Rückfragen auf. Von Luhman kommt die Einsicht, dass je mehr jemand misstraue, desto mehr Informationen fordere er, aber desto weniger vermöge er wiederum diesen Informationen noch zu vertrauen. ${ }^{46}$ Statt einer Rationalisierung der Meinungsbildung, in der, wie John Stuart Mill es sich vorstellte, das Wahre oder zumindest das Richtige sich durch Konfrontation mit möglichst vielen Gegenpositionen herausschält, kommt es dann zu einer Emotionalisierung politischer Einstellungen. Semiöffentliche, private Meinungsquellen im Internet wie etwas Blogs vergrößern das Angebot an Fakten und Meinungen noch, genügen aber oft nicht journalistischen Standards und stiften vielleicht zusätzlich Verwirrung. Eigentlich müsste mit der Informationsmenge auch die Fähigkeit zur Selektion und Verwertung von Informationen steigen. Aber die natürlichen Selektionsmechanismen des Menschen sind eben - die Biologen mögen es bestätigen - nicht auf abstrakte politische Strukturen eingestellt, sondern evolutionsbedingt auf den sozialen Nahraum: Ereignisse dürften mehr Aufmerksamkeit binden als Hintergründe; Bild und Ton mehr als Text; Personen mehr als Positionen; ebenso dürften Informationen, welche mit der persönlichen Weltanschauung harmonieren, präferiert werden.

Angesichts dieser Entwicklungen liegt die Hoffnung auf den klassischen Printmedien, deren Nutzung positiv mit politischem Wissen korreliert. Viel ist von dieser Seite jedoch nicht zu erwarten. Zum einen verlagern die Zeitungen ihr Nachrichtenangebot immer mehr ins Internet und geraten dort in den Sog der Informationsinflation und entsprechender Selektionsmechanismen. Die Konkurrenz zu den visuellen Medien und den Blogs ${ }^{47}$ zwingt sie, sich stärker an den (problematischen) Leseinteressen des Publikums auszurichten und weniger über »Entstehungshintergründe, Ursachen und Folgen von Ereignissen und Entwicklungen $" 48$ zu berichten. Der enorme Aktualitätsdruck entleert das Wort Nachricht seines Sinns; es bleibt immer weniger Zeit, etwas an der Information durch kluge Einordnung zu richten. Sie wird nach der Logik der Transparenz direkt weitergereicht. Zum anderen hat dies auch ökonomische Gründe:49 Zeitungen können immer weniger Werbeeinnahmen generieren, außer durch ihr Online-Angebot, und dadurch fehlen die Mittel, um einen Stab von qualifizierten und fachlich spezialisierten Journalisten zu unterhalten. Während die Politik die Digitalisierung der Gesellschaft an vielen Stellen fördert, bleiben die Zeitungen auf sich gestellt. Das Resultat ist, dass die Medien als ursprünglich »vierte Gewalt « im Staat tendenziell zum Vollstrecker professioneller Polit-PR werden. ${ }^{50}$

46 Vgl. Rzepka 2013, S. 119.

47 Vgl. Sasseen et al. 2013.

48 Rentsch, Mothes 2013, S. 72.

49 Vgl. Blomert 2013.

50 Vgl. Schiffer 2011, S. 27-30.

Leviathan, 42. Jg., 3/2014 


\section{Das Streben nach Authentizität erzeugt Misstrauen}

Die Transparenz des politischen Systems ist das eine, aber das andere ist, dass die Transparenz-Aktivisten auch die Person des Politikers selbst transparent haben möchten. Transparenz ist in Bezug auf die Politikerpersönlichkeit eng mit Authentizität verknüpft. »Authentisch « zu sein bedeutet, dass die Differenz von Schein und Sein verschwindet oder, wie Erving Goffman sagen würde, von der Person und ihrem öffentlichen Theaterspiel. Wenn ein Politiker unerklärte Nebenverdienste hat oder vor verschiedenem Publikum tendenziell Gegensätzliches behauptet, sind seine Motive und Politik intransparent und ermangeln jener kommunikativen Rationalität, die Grundlage unverfälschter demokratischer Diskurse ist. Authentizität kontrastiert mit Lüge und Heuchelei.

Dass Authentizität überhaupt zum Problem werden kann, verdankt sich auch dem Trend zur Personalisierung der Politik (wenngleich man bei dieser These vorsichtig sein sollte: Politik war schon immer akteurszentriert). Diese Personalisierung könnte, wie oben beschrieben, eine Reaktion auf die Informationsinflation sein. Es ist - wiederum aufgrund der evolutionsbiologisch bedingten menschlichen Aufmerksamkeitsökonomie - einfacher, Politik anhand von Personen und ihren charakterlichen Merkmalen wahrzunehmen und einzuordnen denn als inhaltlich komplexen und politisch-administrativ gebrochenen Prozess der Willensbildung. Politik ist natürlich beides, aber wo das öffentliche Interesse sich unverhältnismäßig auf Personen richtet, kann analytisch von Personalisierung gesprochen werden.

Politiker befördern die Personalisierung selbst, indem sie über die sozialen Medien (Twitter, Facebook etc.) direkt mit den Wählern kommunizieren »in einer Weise, die das subjektive Empfinden in den Mittelpunkt stellt $" .{ }^{51}$ Das Bedürfnis danach belegen zum Beispiel Umfragen, welche die Beliebtheit von Politikern bei den Wählern messen. Das Politbarometer mit seinem entsprechenden Ranking gibt es seit 1977, aber seit Beginn der digitalen Revolution wird der Kampf um die oberen Plätze verstärkt über mediale Präsenz ausgefochten, wobei sich das Fernsehen, selbst ein Transparenzmedium, und neue digitale Medien vermischen (indem beispielsweise Fernsehinhalte online abrufbar sind und Talkshow-Auftritte über Twitter kommentiert werden). In den USA fällt auf, dass die Beliebtheit von beziehungsweise das Vertrauen in Politiker systematisch größer ist als das in die Institutionen. Ende November 2013 fiel die Zustimmungsrate für den Kongress auf 9 Prozent, ${ }^{52}$ während die Führer von Senat und Abgeordnetenhaus auf 30-40 Prozent kamen. Bei live übertragenen Kandidaten-Duellen wird dank digitaler Technik in Echtzeit die Zustimmung im Publikum erhoben und dargestellt, wobei eigentlich nur eine psychologische, keine argumentative Wirkung zustande kommen und gemessen werden kann.

Nun geraten diese beiden Tendenzen - die Personalisierung und der Anspruch nach Authentizität - aber leicht in Widerspruch zueinander. Die Personalisierung bewirkt einen Zwang zur Selbstdarstellung, und dies beeinträchtigt jene Sachratio-

51 Zehnpfennig 2013, S. 45.

52 Vgl. Newport 2013. 
nalität, an der sich letztlich Authentizität bemisst. So bemerkte ein externer Sachverständiger der Enquete-Kommission Internet und Gesellschaft, »dass sich die Parlamentarier, sobald die Kameras eingeschaltet waren, teilweise komplett anders als sonst verhielten. Die Gespräche in den Projektgruppen, die nicht gestreamt [also gefilmt] wurden, verliefen anders als die Enquete-Sitzungen, die übertragen wurden $«{ }^{53}$ Vertrauliche Sitzungen waren konstruktiver, in den öffentlichen Sitzungen dagegen wurde parteipolitischer Grabenkampf gespielt.

Die Medien reagieren auf Heuchelei, etwa in der Form politisch motivierter Vernunftbeugung, indem sie die Beobachtung der Politiker intensivieren. So erfreuen sich besonders in den USA seit einigen Jahren sogenannte »Fact Checker « großer Beliebtheit, die den Wahrheitsgehalt und die Konsistenz der Äußerungen von Politikern testen. Große Zeitungen wie die »Washington Post « haben eine entsprechende Rubrik, die Fernsehsender wie CNN und FoxNews haben jeweils ein Segment dazu, und schließlich gibt es Organisationen wie »Fact.Check.org ", die Äußerungen von Politikern auf den Prüfstand stellen und damit zur personenzentrierten Berichterstattung anstiften. In Deutschland setzt die Talkshow Hart aber fair einen Faktencheck ein. Immer werden Personen überprüft, nicht jedoch politische Positionen oder der Output von Institutionen.

Können Lüge und Heuchelei in der Politik tatsächlich unterbunden werden? In der Demokratietheorie ebenso wie in der liberalen Tradition hat der Kampf gegen politische Heuchelei und für eine auf Vernunft basierte Politik eine lange Tradition. Doch heißt dies nicht, dass umfassende Transparenz, die Rhetorik und Politik wieder in Deckung bringt nach dem Modell der Agora, ein sinnvolles Ziel wäre. Der Haupteinwand liegt darin, dass Wahrheit und Authentizität schlicht keine geeigneten Kategorien einer pluralistischen und repräsentativen Demokratie sind.

Ein kursorischer Blick auf diesen besonderen Aspekt der Ideengeschichte mag etwas Orientierung verschaffen. Eine Reihe früherer Denker, angefangen bereits bei Platon und Aristoteles, neigte zu der Auffassung, dass eine politische Elite sich kraft ihrer überlegenen Vernunft nicht an demokratische Mehrheiten binden lassen dürfe. Auch Thomas Hobbes ist ein Vertreter dieser Denkrichtung, insofern für ihn die Herstellung von Frieden und Ordnung der ultimative Maßstab staatlicher Legitimität ist. In dieser Linie unterscheidet der britische Politologe David Runciman ${ }^{54}$ zwei Arten von Aufrichtigkeit. Erstens die Aufrichtigkeit der Politiker in Bezug auf ihre persönlichen Überzeugungen. Diese zu kultivieren sei demokratietheoretisch nicht erstrebenswert, praktisch sogar gefährlich, weil eine aus privaten Überzeugungen schöpfende Politik in Konflikt mit dem Gemeinwohl geraten kann. So habe etwa die Bush-Administration aus ideologischen Gründen den Irak als Feind ausgemacht und unter enormen Kosten an Mensch und Material bekämpft, obwohl dieser gar keine wirkliche Bedrohung für die USA gewesen sei. Wichtiger sei, zweitens, dass Politiker aufrichtig seien in Bezug auf den Umgang mit Macht. Solange ein Politiker die Regeln des demokratischen Systems beachte und verteidige, sei es unerheblich, welche Maske er dabei trage. Ein ähnliches Argument rekonstruiert

53 Enquete-Kommission 2013, S. 26.

54 Runciman 2008.

Leviathan, 42. Jg., 3/2014 
Martin Jay bei dem politischen Philosophen Leo Strauss: ${ }^{55}$ Der erfahrene Politiker wüsste gelegentlich besser, was im aufgeklärten Selbstinteresse des Volkes sei, und dürfe oder müsse daher das Volk auch gezielt täuschen. Ein Beispiel hierfür sei Paul Wolfowitz, der wie andere führende US-Neokonservative in seinem Politik- und Demokratieverständnis maßgeblich von Strauss beeinflusst war. Beispielhaft in einem anderen Sinne sei an der Causa Wolfowitz freilich auch, dass die elitäre Neigung zu einer ideologischen Politik führte, an der selbst eine Dekade später noch keine höhere Vernunft erkennbar ist.

Solche Denkweisen muten daher heute zu Recht problematisch an. Allerdings kennt auch das Grundgesetz Transparenz-Schranken, die einen ähnlichen Geist atmen. Das Gewissen der Abgeordneten ist durch das Grundgesetz aus Gründen der Sicherung gegenüber der Volatilität der öffentlichen Meinung geschützt: Die Abgeordneten "sind Vertreter des ganzen Volkes, an Aufträge und Weisungen nicht gebunden und nur ihrem Gewissen unterworfen «. ${ }^{56}$ Dabei wird offensichtlich auf das "gute « Hobbes'sche Gewissen der systemerhaltenden Art vertraut. Im Übrigen geht auch der moderne Liberalismus stillschweigend davon aus, dass bestimmte Rechte wie das Recht auf Freiheit, Leben und Eigentum so grundlegend sind, dass sie der demokratischen Meinungsbildung entzogen sein sollten. Zu viel Transparenz, ein permanenter Offenlegungs- und Rechtfertigungszwang, könnte den Spielraum für die Ausübung des Gewissens beschränken und damit im Übrigen auch jene Kompromissfähigkeit beschädigen, welche der demokratische Prozess erfordert.

Die Enquete-Kommission Internet und Gesellschaft ist ein Beispiel genau dafür: Sie rühmt sich, dass ihre Arbeit »in einem Maß öffentlich dokumentiert, erläutert und damit im eigentlichen Sinne transparent gemacht wurde, wie es bisher in keinem anderen Gremium des Deutschen Bundestages erfolgte «. ${ }^{57}$ Allerdings hat sie auch zu keinen Ergebnissen geführt: Im Abschlussbericht »Datenschutz, Persönlichkeitsrechte « ${ }^{58}$ kommen auf 54 Empfehlungen (39 Seiten) 28 Sondervoten (26 Seiten) der Oppositionsparteien und Sachverständigen. Ein Kompromiss wäre für den Schutz der Grundrechte im digitalen Zeitalter, konkret des Rechts auf informationelle Selbstbestimmung, besser gewesen als die Blockade durch eine Verhärtung der politischen Fronten. ${ }^{59}$

Man kann den Fall aber auch etwas anders betrachten. Eine entsprechend pragmatischere Sicht auf die »demokratische Lüge « findet Jay bei Theodor Adorno und Hannah Arendt. Für Adorno sei die vollständige Transparenz der Sprache, auf die unter anderem Bentham so viel Wert legte, ${ }^{60}$ verdächtig, weil sie eine politische Einheit und sachliche Klarheit suggeriere, oft massenmedial vermittelt, wie sie in der pluralistischen Demokratie eigentlich gar nicht möglich seien. Das Streben nach

55 Jay 2004, S. 58-59.

56 Artikel 38 des Grundgesetzes.

57 Enquete-Kommission 2013, S. 8.

58 Enquete-Kommission 2012.

59 Vgl. Finel 1999 für ein ähnliches Argument in Bezug auf internationale Diplomatie.

60 Runciman 2008, S. 116-141. 
ultimativer Klarheit könne auch in Xenophobie und andere Extremismen umschlagen. Das, was tatsächlich vernünftig sei, lasse sich eigentlich nur ambivalent ausdrücken. ${ }^{61}$ Hannah Arendt habe dagegen versucht, Politik und Philosophie strikt zu trennen. Politik wirke auf eine Zukunft hin, und deshalb könne jene Rhetorik, die helfe, einen avisierten zukünftigen Zustand zu erreichen, nicht an der Gegenwart und ihren Fakten gemessen werden: »In dem Maße, in dem Politik der möglichen Zukunft dient, auf Meinungen eher als auf harten Tatsachen beruht, sich in Kontingenz statt ewigen Wahrheiten bewegt, Rhetorik eher als deduktive Logik bemüht, und auf Pluralität mehr als auf Einheit beruht, kann das Lügen aus ihren Vorhaben nicht völlig ausgeschlossen werden «. ${ }^{62}$

Das gilt besonders für die Demokratie, zumal die repräsentative Regierungsform. Sie beruht auf dem Wettkampf um Macht und der regelmäßigen Ablösung einer Regierung durch die Opposition, was nur über die Kritik an der Regierung und die Artikulation alternativer Ideen gelingt. Es sind also im demokratischen System immer mindestens zwei Perspektiven im Umlauf, die logischerweise nicht beide gleichzeitig »wahr « sein können. Ihre Geltung hängt davon ab, wie sie beworben werden, und dabei wäre zu viel Aufrichtigkeit ein Hindernis. »Das Paradox liberaler Demokratien besteht darin, dass sie Heuchelei begünstigen, weil die Politik der Überzeugung von den beteiligten Sprechern [...] gelegentlich ein gewisses Maß an Verstellung erfordert. «63 Dass die Enquete-Kommission zu keinem Beschluss kam, könnte so gesehen auch schlicht daran liegen, dass sich noch keine gesellschaftliche Mehrheit zu ihrem Thema gebildet hat, die Grundlage für einen Kompromiss hätte sein können.

Damit ist noch keine direkte Antwort gegeben auf das Problem, wie die legitime Heuchelei, die Teil des demokratischen Spiels ist, von der gefährlichen Lüge unterschieden werden kann, welche der Machtanmaßung dient und das Spiel insgesamt gefährdet. Hätte man den Irak-Krieg, der mit fingierten Gründen begonnen wurde, verhindern können durch mehr Transparenz? Hätte mehr Transparenz den NSASkandal beziehungsweise die massenhafte, rechtswidrige Ausspähung unbescholtener Bürger verhindern oder beenden können? Die demokratische Kontrolle hat in beiden Fällen offensichtlich ebenso versagt wie das demokratische Gewissen der Beteiligten. Die Antwort könnte allerdings auch darin liegen, das politische Spiel der Demokratie mit etwas mehr Gelassenheit zu betrachten und auf seine Selbstreinigungskräfte zu vertrauen. Nicht jede Art von politischer Fehlleistung muss präventiv-institutionell verhindert werden. Es zeichnet insbesondere Demokratien aus, dass vieles letztlich doch an die Öffentlichkeit kommt und damit die in allen Gesellschaftsformen vorhandenen versteckten Informationssysteme und deren Missbrauch nicht überhandnehmen. Whistleblower, investigativer Journalismus, Interessengruppen etc. spielen dabei eine wichtige Rolle. Es wäre besser, auf einen solchen demokratischen Pluralismus der nachgängigen Rechenschaftspflicht zu ver-

\author{
61 Jay 2004, S. 60. \\ 62 Ebd., S. 62. \\ 63 Shklar 2014, S. 60.
}

Leviathan, 42. Jg., 3/2014 
trauen, als den politischen Prozess nach ästhetischen Kriterien von Wahrheit und Authentizität neu einrichten zu wollen.

\section{Machtverschiebungen, aber nicht zugunsten der Demokratie}

In den vorherigen Abschnitten haben wir gesehen, dass die Bürger ihrer eigenen Rolle nicht gerecht werden, weil sie Information nicht in politisches Urteil überführen können, und dass sich auch in Bezug auf die Rolle der Politik, die notwendig taktisch verfährt, kein neuartiges Vertrauen einstellen wird. Die so vorbereitete These einer paradoxen Wirkung von Transparenz soll nun unter dem entscheidenden Aspekt von Macht ins Ziel geführt werden. Rekapitulieren wir für einen Augenblick: »Wissen ist Macht «, so die Prämisse der Transparenz-Aktivisten, und daher bewirke die Informationsgesellschaft, dass Politik wirklich "Sache der Bürger « wird. Offensichtlich werden hier Information und politisches Urteil in einen Topf geworfen, aber darin erschöpft sich die Transparenz-Kritik noch lange nicht. Transparenz-Aktivisten scheinen jedenfalls sehr auf die Gleichung »Information = Macht « fokussiert zu sein: Wäre nur der Staat offen und der Bürger informiert, würde sich daraus wie von selbst eine Verbesserung der Demokratie ergeben.

Dieser Gedankengang beruht auf einer Ausblendung der Komplexitäten des politischen Prozesses. Wie genau soll sich Information im Verbund mit Partizipation in politische Entscheidungen umsetzen? Und kann Macht durch frei verfügbare Information nicht auch andere Akteure als den Bürger begünstigen, etwa Regierungen und Interessenorganisationen? Betrachten wir der Reihe nach diese Akteure und wie sich ihre politischen Rollen infolge der Transparenz(über)steigerung faktisch verändern.

Die demokratische Macht der Bürger kann sich auf zwei Arten äußern: in Gestalt periodischer Wahlen und durch eine gesteigerte, fall- und interessenspezifisch zustande kommende (Online-)Partizipation. Eine Steigerung der Wahlkompetenz durch Transparenz könnte nur unterstellt werden, wenn ein Mehr an Information zu einem besseren politischen Urteil führt. Wahlomaten könnten dabei hilfreich sein, ansonsten gilt aber, was oben zur informationellen Überforderung gesagt wurde (vgl. Kapitel 3). Vor allem ist jedoch die Einflussnahme über direkte Beteiligung zu prüfen, auf welche Transparenz-Aktivisten pochen. Hier klafft eine bemerkenswerte Lücke zwischen dem, was als neuartige politische Aktivität ausgegeben wird, und der mutmaßlichen politischen Wirksamkeit. Das vermehrte Konsumieren von Nachrichten, die Online-Kommunikation mit Politikern und der Leserbrief etwa gelten als Aktivitäten, die eine politische Belebung anzeigen. ${ }^{64}$ Jedoch können diese Aktivitäten eher mit dem erleichterten Zugang zur Öffentlichkeit erklärt werden, wie er sich dem "Stubensurfer « durch das Internet eröffnet, als mit neuem politischen Interesse. ${ }^{65}$ Diese Form der politischen Beteiligung direkt aus dem Privaten heraus führt meist nicht zum »Aufbau handlungsfähiger kollektiver Aktions-

64 Emmer 2013, S. 149.

65 Geser 2011, S. 9. 
formen « 66 und bleibt daher für die Politik häufig bedeutungslos: Es gibt keinerlei Anhaltspunkte, dass Politiker Bürgereingaben im Rahmen der direkten Beteiligung - etwa durch die in diesem Zusammenhang gelobten sozialen Netzwerke, im Rahmen von liquid democracy-Verfahren oder eines Online-Dialogs ${ }^{67}$ - tatsächlich in einer bedeutungsvollen Weise berücksichtigen. Wie sollten sie auch: Der Aufwand, alles zu lesen und zu beantworten, wäre durch die Zunahme von Online-Beteiligungsverfahren enorm. Da außerdem die verstreut eingefangenen Kommentare und Voten wechselhaften Themenzyklen folgen, wäre es politisch leichtsinnig, sich daran zu orientieren. Und weil sie meist nicht repräsentativ sind, würde es zudem an demokratische Verantwortungslosigkeit grenzen, sich davon leiten zu lassen.

Dies lässt eigentlich nur zwei Funktionen der online hergestellten Partizipation zu: Sie sorgt vielleicht nur für emotionale Entlastung und gibt das Gefühl, gehört zu werden, ${ }^{68}$ ist als Kommunikationsakt aber so wirkungslos wie das Freisetzen einer Flaschenpost. Der andere Fall wäre der online bewirkte Proteststurm, der tatsächlich politischen Einfluss entfaltet. Die Aussetzung des ACTA-Abkommens durch den Netzprotest wäre hier einschlägig oder die Absetzung von Ministern, die über Internet-Aktivitäten des Plagiats überführt wurden. Beides sind neue Phänomene. Nur: In solchen eher spontanen Aktionen äußert sich, um eine Differenzierung von Habermas aufzugreifen, der Bürger weniger als » rationale«, sondern mehr als " personale Autorität « ${ }^{69}$ Nicht die Vernunft bewegt ihn, sondern im besten Fall das vernünftige Gefühl. Der Bürger ist nicht das demokratische Subjekt, das entsprechend des Konzepts der liberalen Öffentlichkeit Themen auf die Agenda setzt, sondern er entfaltet lediglich prohibitive Wirkung.

Diese Situation verstärkt ein ohnehin vorhandenes strukturelles Ungleichgewicht: Umfang und Aktivismus des politischen Diskurses explodieren förmlich, aber die Politik bleibt davon - außer im Fall des gelegentlichen Skandals - merkwürdig unberührt. Mehr noch: Die öffentliche Meinung fragmentiert sich, wird dadurch unverbindlich und eröffnet der Politik neuen Handlungsspielraum, sofern es ihr gelingt, den demokratischen Schein der Volksnähe zu wahren. Das ist eine direkte Folge des information overload, wie Davis argumentiert: Wenn Informationen von jedem über alles vorliegen, entsteht ein Konkurrenzkampf um deren Durchsetzung. "Am Ende wird das Publikum zu jenen Quellen neigen, die in der Lage sind, einen solchen Konkurrenzkampf zu gewinnen. «70 Das sind in der Regel nicht die Philosophen, sondern der Staat und organisierte Verbände.

Bereits eine anekdotische Bestandsaufnahme des politischen Diskurses im Zeitalter der Transparenz fördert massive Kommunikations- und damit, jedenfalls im Modell der Agora, demokratische Beziehungsstörungen von Regierung und Volk zutage. Habermas unterscheidet bekanntlich, wie bereits angesprochen, die drei

66 Ebd.

67 Pernice 2013, S. 13-14.

68 Plake et al. 2001, S. 86.

69 Habermas 1973, S. 12.

70 Davis 1999, S. 7. 
»Geltungsansprüche" Wahrheit, Aufrichtigkeit, Angemessenheit, welche jene Art gelingender rationaler Kommunikation kennzeichnen, auf die auch TransparenzAktivisten hinarbeiten. An ihnen kann die Güte der politischen Kommunikation gemessen werden, um die es, wie eine kursorische Betrachtung zeigt, nicht gut steht. Objektive Wabrheit: In den USA sind die offene Leugnung des Klimawandels, der Evolution und der Folgen eines libertären Waffenrechts etc. Positionen, mit denen Bundespolitik betrieben wird, obwohl sie in der Öffentlichkeit als objektiv falsch bekannt sind. Subjektive Aufrichtigkeit: Die CDU/CSU und FDP haben sich in der letzten Legislaturperiode öffentlich für Datenschutz ausgesprochen und in diesem Zusammenhang auch die erwähnte Enquete-Kommission eingerichtet, die als Fortschritt bei der Schaffung eines neuen Standards der Bürgerbeteiligung gesehen wurde. ${ }^{71}$ Gleichzeitig hat die damalige Bundesregierung in Deutschland sowie auf EU-Ebene Reformen verhindert und sogar an der Aufweichung existierender Regulierung gearbeitet. ${ }^{72}$ Normative Angemessenheit: Nach nahezu einhelliger Auffassung von Datenschützern, von Verfassungsjuristen und mehrheitlich auch der Bevölkerung verstoßen bestimmte geheimdienstliche Praktiken, insbesondere die Massenausspähung, der Einsatz bewaffneter Drohnen, Verhörtechniken etc., gegen Verfassungsnormen. Obgleich im Prinzip schon früh öffentlich bekannt, hatte diese Information keine politische Konsequenzen. ${ }^{73}$

Für Roberts ist diese gestörte Beziehung zwischen Volk und Regierung ein Ausdruck dafür, dass die Menschen ihre demokratische Mitverantwortung aufgegeben hätten. ${ }^{74}$ Die Regierung werde, obgleich gewählt, gar nicht mehr als Teil des demokratischen Gemeinwesens begriffen. Roberts erklärt nicht, wie es zu dieser »Ethik des Desengagements (ethic of detachment) «75 kam. Vielleicht ist $z u$ wenig Transparenz der Grund dafür. Meine Vermutung wäre: Die Politik ist - unter Bedingungen der Transparenz - zur aktiven Öffentlichkeitsarbeit gezwungen, sie stellt Öffentlichkeit bedarfsgerecht selbst her, statt sich nach einer vorgefundenen Öffentlichkeit zu richten. Die demokratische Wirkungsrichtung dreht sich damit um, das Elektorat und der einzelne Bürger werden vom Subjekt zum Objekt der Politik, und dies unterbindet eine positive Selbstidentifikation mit dem Handeln der Regierung.

Mit dieser These lassen sich aktuelle Befunde der Forschung einordnen. So wird etwa argumentiert, dass die Politik unter medialer Dauerbeobachtung in den Modus einer Dauerkampagne gerät und darauf mit einer Zentralisierung reagiert $;{ }^{76}$ politische Macht und die Pressearbeit werden in der Regierung konzentriert, statt subsidiär auf untere Ebenen verlagert zu werden, was die Regierung schlagkräftiger, aber

71 Vgl. Pernice 2013, S. 13.

72 Baumann 2013.

73 Vgl. Roberts 2006 a, S. 231 ff.: So seien in den USA die Foltervorwürfe schon in der ersten Amtszeit von Präsident Bush bekannt geworden, sie hätten aber im Wahlkampf schlicht keine Rolle gespielt, sodass es zur Wiederwahl eines Präsidenten kam, dessen Administration grobe verfassungsrechtliche Verstöße zur Last gelegt werden.

74 Ebd., S. 231-238.

75 Ebd., S. 237.

76 Heald 2006, S. 63. 
eben auch undemokratischer macht. Zweitens werden Entscheidungen in einer durch den Takt der digitalen Öffentlichkeit » beschleunigten Demokratie « in informelle Runden verlagert und entziehen sich damit der parlamentarischen Kontrolle. ${ }^{77}$ Drittens wird, je stärker die Politik die Öffentlichkeit und ihre wechselnden Mehrheiten in Rechnung stellen muss, die Gegenbeobachtung des Wählers intensiviert. Wenn aber die politischen Systeme "auf Impulse der Veränderung eingestellt sind und diese sogar in ihren Planungen vorwegnehmen, wenn sie vielleicht sogar initiativ werden [...], ist möglicherweise auch nicht mehr zu entscheiden, wer wen beeinflusst « ${ }^{78}$ Es fängt damit an, dass Informationsfreiheits- und TransparenzGesetze der Regierung gewisse Spielräume lassen, welche Informationen wie aufbereitet der Öffentlichkeit zugänglich gemacht werden, und es endet damit, dass eine aus demoskopischen Gründen erfolgversprechende politische Position als "alternativlos « (Kanzlerin Merkel) erklärt wird, ohne sie einer gesellschaftlichen Willensbildung auszusetzen.

Bleibt die Rolle der organisierten Interessen. Sie haben, was der durchschnittliche Bürger nicht hat: das Interesse, die Ausdauer und die Fähigkeit, Informationen zu verarbeiten, big data ebenso wie small data, und - zum Beispiel in Gestalt von Lobbyismus - in politischen Einfluss umzusetzen. »Nicht der normale Bürger ist es, der nur darauf wartet, endlich ein staatliches Register durchkämmen zu dürfen, sondern professionelle Beobachter von Regierung und Verwaltung sind es, die hier ein zusätzliches Instrument in die Hand bekommen. «79 Wie die Regierung, aber ohne demokratische Legitimation, können Interessenorganisationen somit Öffentlichkeit unverhältnismäßig stark beeinflussen. ${ }^{80}$ Dass gut finanzierte Gruppen im Internet stärker mobilisieren können, war bereits früh nachweisbar. ${ }^{81}$ Wer eine Politik im Modus der Publizität fordert, muss daher wissen, in welche Löwengrube er sich begibt. Bedenkt man, dass wirtschaftliche Verbände in der Regel eine höhere Finanzund Durchsetzungskraft haben als solche aus dem Bereich Sozialwesen und Kultur, gibt dies Anlass zur Sorge, dass die »Kampfparität « 82 eines pluralistischen Gemeinwesens in Schieflage gerät. Besonders jene intermediären Verbände, die selbst einen demokratischen Aufbau und folglich auch ein höheres Maß an Legitimität haben (etwa Parteien und Gewerkschaften), könnten durch die (vermeintliche) digitale Ermächtigung des Bürgers an Bedeutung verlieren.

\section{Resümee}

Es wurde argumentiert, dass die Transparenz-Aktivisten auf ein illusorisches Politikmodell hinsteuern und dabei die demokratisierende Kraft von Transparenz über-

77 Korte 2012.

78 Plake et al. 2001, S. 187.

79 Wewer 2014, S. 12.

80 Heald 2006, S. 62.

81 Davis 1999, S. 81-83.

82 Schmidt 2010, S. 219.

Leviathan, 42. Jg., 3/2014 
schätzen. Transparenz ist nicht jenes Heilmittel, welches die (Rest-)Mängel der Demokratie beseitigt und diese durch direktdemokratische Elemente belebt. Es kommt vielmehr zu einer Anverwandlung der Transparenz durch das politische System und zu verschiedenen nichtintendierten Effekten, was letztlich die herrschaftspolitische Balance der modernen Repräsentativdemokratie in Schieflage zu bringen droht: Der Bürger ist von der ihm zugewiesenen Rolle überfordert, weil er Informationen nicht angemessen verarbeiten kann; der Politiker wird in einem demokratischen System niemals völlig transparent und authentisch erscheinen (und wo er es doch tut, wäre besonders kritisch zu fragen, ob dies Ausdruck von politischer Exzellenz oder Schauspielerei wäre); und am Ende profitieren vor allem die organisierten Akteure, das heißt die Regierung und die Verbände, welche gewiefter sind im Umgang mit der Öffentlichkeit.

Dies bedeutet nicht, dass das Internet und die von ihm bereitgestellte Transparenz völlig wirkungslos sind. Ein Mindestmaß an demokratischer Transparenz ist unabdingbar, und durch die Offenlegung von staatlichem Fehlverhalten, etwa durch WikiLeaks, sowie die Möglichkeit von Online-Vernetzung infolge von Informationsimpulsen entsteht ein beachtliches Mobilisierungspotenzial. Aber solche Proteste tragen wenig zu einer Optimierung der Politik nach den Maßstäben von Vernunft und demokratischer Selbstbestimmung bei. Indem Transparenz-Aktivisten ihre Energie auf die Politik lenken, fördern sie vielleicht jene Entfremdung der Politik von den Bürgern, die ihnen dann zum Problem wird. Um Politik wieder stärker zu einer »Sache der Bürger « zu machen, müssten erst - oder zumindest im Gleichtakt mit der Implementation von Transparenz - das Interesse und die Politikfähigkeit der Bürger gestärkt werden. Das ist kein einfaches Unterfangen.

\section{Literatur}

Baumann, Max-Otto 2013. »Datenschutz im Web 2.0. Der Politische Diskurs über Privatsphäre in Sozialen Netzwerken", in Im Sog des Internets, hrsg. v. Ackermann, Ulrike, S. 15-52. Frankfurt a. M.: Humanities Online.

Benhabib, Seyla 1996. Democracy and difference. Contesting the boundaries of the political. Princeton: Princeton University Press.

Blomert, Reinhard 2013. »Die gefährdete Demokratie. Überlegungen zur schleichenden Aushöhlung der Pressefreiheit ", in Blätter für deutsche und internationale Politik 1, S. 97-100.

Davis, Richard 1999. The web of politics. The internet's impact on the American political system. Oxford: Oxford University Press.

Egger de Campo, Marianne 2014. »Neue Medien - alte Greedy Institutions «, in Leviathan 42, 1, S. 7-28.

Emmer, Martin 2013. »Evolution oder Revolution? Der Einfluss des Internets auf die Rolle der Bürger in der Politik ", in Politische Kommunikation in Zeiten des Medienwandels, hrsg. v. Roessing, Thomas; Podschuweit, Nicole, S. 137-161. Berlin: De Gruyter.

Enquete-Kommission Internet und Digitale Gesellschaft 2012. Fünfter Zwischenbericht. Datenschutz, Persönlichkeitsrechte. Deutscher Bundestag, Drucksache 17/8999. Berlin.

Enquete-Kommission Internet und Digitale Gesellschaft 2013. Schlussbericht. Deutscher Bundestag, Drucksache 17/12550. Berlin.

Finel, Bernard I.; Lord, Kristin M. 1999. »The surprising logic of transparency ", in International Studies Quarterly 43, S. 315-339.

Geser, Hans 2011. Kakophonie und Selbstorganisation in der digitalen Agora. http://geser.net/ intcom/t_hgeser23.pdf (Zugriff vom 14.07.2014). 
Habermas, Jürgen 1962. Strukturwandel der Öffentlichkeit. Untersuchungen zu einer Kategorie der bürgerlichen Gesellschaft. Neuwied: Luchterhand.

Habermas, Jürgen 1973. Kultur und Kritik. Verstreute Aufsätze. Frankfurt a. M.: Suhrkamp.

Habermas, Jürgen 1992. Faktizität und Geltung. Beiträge zur Diskurstheorie des Rechts und des demokratischen Rechtsstaats. Frankfurt a. M.: Suhrkamp.

Han, Byung-Chul 2012. Transparenzgesellschaft. Berlin: Matthes \& Seitz.

Heald, David 2006. "Transparency as an instrumental value ", in Transparency. The key to better governance?, hrsg. v. Hood, Christopher; Heald, David. S. 59-73. Oxford: Oxford University Press.

Hood, Christopher; Heald, David. Hrsg. 2006. Transparency. The key to better governance? Oxford: Oxford University Press.

Jay, Martin 2004. »The ambivalent virtues of mendacity «, in Index of Censorship 33, 2, S. 54-65.

Kamps, Klaus 2000. »Die >Agora des Internets. Zur Debatte politischer Öffentlichkeit und Partizipation im Netz «, in Zerfall der Öffentlichkeit?, hrsg. v. Jarren, Otfried; Imhof, Kurt; Blum, Roger, S. 227-239. Wiesbaden: Westdeutscher Verlag.

Kneuer, Marianne 2013. »Bereicherung oder Stressfaktor? Überlegungen zur Wirkung des Internets auf die Demokratie", in Das Internet: Bereicherung oder Stressfaktor für die Demokratie?, hrsg. v. Kneuer, Marianne, S. 7-31. Baden-Baden: Nomos.

König, Tim 2012. In guter Gesellschaft? Einführung in die politische Soziologie von Jürgen Habermas und Niklas Luhmann. Wiesbaden: VS Verlag für Sozialwissenschaften.

Korte, Karl-Rudolf 2012. »Beschleunigte Demokratie: Entscheidungsstress als Regelfall «, in Aus Politik und Zeitgeschichte 62, 7, S. 21-26.

Leggewie, Claus; Maar, Christa. Hrsg. 1998. Internet \& Politik. Von der Zuschauer-zur Beteiligungsdemokratie? Köln: Bollmann.

Lord, Kristin M. 2006. The perils and promise of global transparency. Why the information revolution may not lead to security, democracy, or peace. New York: State University of New York.

Maier, Jürgen; Bathelt, Severin 2013. „Unbekanntes Europa? Eine vergleichende Analyse zu Verteilung und Determinanten von Kenntnissen über die Europäische Union ", in Zivile Bürgergesellschaft und Demokratie. Aktuelle Ergebnisse der empirischen Politikforschung, hrsg. v. Keil, Silk; Thaidigsmann, Isabell, S. 413-432. Wiesbaden: Springer VS.

Manent, Pierre 2014. »The crisis of liberalism ", in Journal of Democracy 25, 1, S. 131-141.

Mill, John Stuart 2010 [1859]. Über die Freiheit. Stuttgart: Reclam.

Mill, John Stuart 2013 [1861]. Betrachtungen über die Repräsentativregierung. Frankfurt a. M.: Suhrkamp.

Mouffe, Chantal 2007. Über das Politische. Wider die kosmopolitische Illusion. Frankfurt a. M.: Suhrkamp.

Newport, Frank 2013. »Congressional approval sinks to record low «, in GALLUP politics. www.gallup.com/poll/165809/congressional-approval-sinks-record-low.aspx (Zugriff vom 29.01.2014).

Oelze, Berthold 2013. "Unbehagen an der Politik. Zum neuen Strukturwandel der Öffentlichkeit ", in Der entmachtete Leviathan. Löst sich der souveräne Staat auf?, hrsg. v. Bach, Maurizio, S. 247-259. Baden-Baden: Nomos.

O'Neill, Onora 2002. A question of trust. The BBC Reith lectures 2002. Cambridge: Cambridge University Press.

Pernice, Ingolf 2013. Informationsgesellschaft und Politik. Vom neuen Strukturwandel der Öffentlichkeit zur Global Policy Governance. HIIG Discussion Paper Series No. 2013-02. Berlin: Alexander von Humboldt Institut für Internet und Gesellschaft.

Plake, Klaus; Jansen, Daniel; Schuhmacher, Birgit 2001. Öffentlichkeit und Gegenöffentlichkeit im Internet. Politische Potenziale der Medienentwicklung. Wiesbaden: Westdeutscher Verlag.

Rentsch, Mathias; Mothes, Cornelia 2013. "Journalismus und Selbstfindung ", in Politische Kommunikation in Zeiten des Medienwandels, hrsg. v. Roessing, Thomas; Podschuweit, Nicole, S. 71-99. Berlin: De Gruyter.

Roberts, Alasdair 2006 a. Blacked out. Government secrecy in the information age. Cambridge: Cambridge University Press. 
Roberts, Alasdair 2006 b. »Dashed expectations: governmental adaptation to transparency rules ", in Transparency. The key to better governance?, hrsg. v. Hood, Christopher; Heald, David, S. 107-126. Oxford: Oxford University Press.

Runciman, David 2008. Political hypocrisy. The mask of power, from Hobbes to Orwell and beyond. Princeton: Princeton University Press.

Rzepka, Vincent 2013. Die Ordnung der Transparenz. Jeremy Bentham und die Genealogie einer demokratischen Norm. Münster: LIT Verlag.

Sartori, Giovanni 1992. Demokratietheorie. Darmstadt: Wissenschaftliche Buchgesellschaft.

Sasseen, Jane; Olmstead, Kenny; Mitchell, Amy 2013. »Digital: as mobile grows rapidly, the pressures on news intensify ", in The state of new media 2013. Pew Research Center. http:// stateofthemedia.org/2013/digital-as-mobile-grows-rapidly-the-pressures-on-news-intensify/ (Zugriff vom 29.01.2014).

Schiffer, Sabine 2011. »Informationsmedien in der Postdemokratie. Zur Bedeutung von Medienkompetenz für eine lebendige Demokratie", in Aus Politik und Zeitgeschichte 1-2/2011, S. 27-32.

Schmidt, Manfred G. 2010. Demokratietheorien. Wiesbaden: VS Verlag für Sozialwissenschaften.

Schremmer, Eckart 1994. Steuern und Staatsfinanzen während der Industrialisierung Europas. England, Frankreich, Preussen und das Deutsche Reich 1800 bis 1914. Heidelberg: Springer.

Shane, Peter M. 2004. »The electronic federalist: the internet and the eclectic institutionalization of democratic legitimacy ", in Democracy online. The prospects for political renewal through the internet, hrsg. v. Shane, Peter M., S. 65-82. New York: Routledge.

Shklar, Judith N. 2014 [1985]. Ganz normale Laster. Berlin: Matthes \& Seitz.

Sztompka, Piotr 2010. »Does democracy need trust, or distrust, or both? «, in Transparenz. Multidisziplinäre Durchsichten durch Phänomene und Theorien des Undurchsichtigen, hrsg. v. Jansen, Stephan A.; Schröter, Eckhard; Stehr, Nico, S. 84-291. Wiesbaden: VS Verlag für Sozialwissenschaften.

Volk, Christian 2013. "Zwischen Entpolitisierung und Radikalisierung - Zur Theorie von Demokratie und Politik in Zeiten des Widerstands ", in Politische Vierteljahresschrift 54, 1, S. 75-110.

Wewer, Göttrik 2014. »Allheilmittel Transparenz? Anmerkungen zur Diskussion «, in Verwaltung \& Management 20, S. 4-18.

Witschge, Tamara 2004. "Online deliberation: possibilities of the internet for deliberative democracy ", in Democracy online. The prospects for political renewal through the internet, hrsg. v. Shane, Peter M., S. 109-122. New York: Routledge.

Young, Iris Marion 1996. "Communication and the other: beyond deliberative democracy ", in Democracy and difference. Contesting the boundaries of the political, hrsg. v. Benhabib, Seyla, S. 120-135. Princeton: Princeton University Press.

Zehnpfennig, Barbara 2013. "Mehr Transparenz - weniger Demokratie? Über die politische Dimension des Internets ", in Das Internet: Bereicherung oder Stressfaktor für die Demokratie?, hrsg. v. Kneuer, Marianne, S. 35-56. Baden-Baden: Nomos. 
Zusammenfassung: Der Aufsatz ordnet die seit einigen Jahren mit Vehemenz vorgetragene Forderung nach mehr Transparenz in der Politik kritisch und im Rückgriff auf die politische Theorie ein. Es wird argumentiert, dass Transparenz eine harmonische Vorstellung der Politik nach dem Modell der griechischen Agora und der liberalen Öffentlichkeit impliziert, die nicht mit dem System der modernen repräsentativen Demokratie kompatibel ist. Transparenz überfordert den Bürger, verkennt die Bedeutung von Rhetorik und begünstigt letztlich Regierungen und organisierte Interessen. Ein Übermaß an Transparenz könnte der Demokratie schaden.

Stichworte: Transparenz, Internet, Demokratie, Heuchelei, Bürger, Macht

\section{The beautiful norm of transparency and the beast of politics: unintended consequences of the new ideology of publicity}

Summary: The article evaluates the vehement advocacy for more political transparency which has been propagated for several years through the lense of political theory. It argues that transparency implies a harmonious model of the political system similar to the Greek agora which is ill-conceived and incompatible with the reality of our modern representative democracy. Too much transparency might harm democracy.

Keywords: transparency, democracy, information, truth, power, citizens' role, hypocrisy

\section{Autor}

Max-Otto Baumann, M.A.

John Stuart Mill Institut für Freiheitsforschung

Ludwig-Guttmann-Straße 6

69123 Heidelberg

max.baumann@hochschule-heidelberg.de 